\title{
Progress on the Development of an Integrated Canopy Fluorescence Model
}

\author{
John R. Miller ${ }^{1}$, Michael Berger ${ }^{2}$, Luis Alonso ${ }^{3}$, Zoran Cerovic ${ }^{4}$, Yves Goulas ${ }^{4}$, Stéphane Jacquemoud ${ }^{5}$, Juliette \\ Louis $^{4}$, Gina Mohammed ${ }^{6}$, Ismael Moya ${ }^{4}$, Roberto Pedros ${ }^{4}$, José F. Moreno ${ }^{3}$, Wout Verhoef ${ }^{7}$, Pablo J. Zarco-Tejada ${ }^{8}$ \\ 1 Department of Physics and Astronomy, York University, Toronto, Canada - jrmiller@yorku.ca \\ 2 European Space Agency/ESTEC, Noodwijk, The Netherlands - Michael.Berger@esa.int \\ ${ }^{3}$ Department of Physics, University of Valencia, Valencia, Spain \\ ${ }^{4}$ LURE/CNRS, Université Paris 11, Orsay, France \\ ${ }^{5}$ LED, Université Paris 7, Paris, France \\ ${ }^{6}$ P \& M Technologies, Sault Ste. Marie, ON, Canada \\ ${ }^{7}$ National Aerospace Laboratory NLR - The Netherlands \\ ${ }^{8}$ Grupo de Óptica Atmosférica (GOA-UVA), Universidad de Valladolid, Spain
}

\begin{abstract}
Typical environmental plant stress factors are excess of light, deficiencies of water and nutrients, temperature extremes, diseases, pests and pollutants. An early indicator for vegetation status and vitality by means of remote sensing would therefore serve a range of applications such as renewable resource management and precision farming. Vegetation fluorescence is a direct indicator for plant physiology, and could therefore be used as an early indicator for vegetation health status and vitality. Vegetation chlorophyll fluorescence is a function of photochemical processes and efficiency, which are directly linked to primary productivity and $\mathrm{CO}_{2}$ flux from the atmosphere, and could therefore also provide a means to assess the terrestrial carbon cycle.
\end{abstract}

A study was launched in October 2002 by the European Space Agency to advance the underlying science of a possible future vegetation fluorescence space mission by addressing the need for an integrated canopy fluorescence model. The objective of this study is to review and advance existing fluorescence models at the leaf level and to integrate these into canopy models in order to simulate the combined spectral reflected radiance and passive fluorescence emission signals. This model is to be validated with new and existing field campaign measurements.

This paper reports on the status of this project. The input radiometric and photosynthetic variables have been selected to define the vegetation fluorescence signal consisting of far-red and red chlorophyll fluorescence as spectral emission features, normalized to the canopy illumination levels, when linked to the leaf-level fluorescence reflectance-transmittance model defined in this study. Measurement protocols to validate fluorescence-leaf models will be defined.

Keywords-solar-induced fluorescence, modelling, vegetation

\section{INTRODUCTION}

It is now recognized that terrestrial vegetation systems, and biosphere processes and interactions are so complex that detailed characterization and description of the system is needed and that model development needs a very precise physical basis. As a consequence, remote sensing requires development of accurate quantitative approaches that exploits all types of information in the optical signal. However, with respect to biosphere dynamics, notably within a short time scale, spectral reflectance and directionality of radiance cannot alone provide complete information since these responses are not directly connected to fundamental processes of plant physiology.

This connection is found however in vegetation fluorescence, known to convey very specific information related to the efficiency of utilization of light energy by the plant, thus related to plant vitality and to biomass production. This would open new ways for global monitoring of vegetation, with improved estimates of vegetation photosynthetic activity and direct implications for surface carbon flux estimation. Because active Chl fluorescence remote sensing from a satellite is considered far beyond current technical possibilities [1], attention is currently focused on passive Chl fluorescence remote sensing methods. Hence, the FLEX (Fluorescence Explorer) mission was proposed to ESA in 1998 to explore the possible use of the Fraunhofer lines of the solar spectrum for passive measurements of natural sunlight-induced fluorescence. The FLEX-Instrument definition pre-study as well as a review of the scientific requirements and instrumental requirements, all under ESA contract, have recently been reported [2,3]. However, there are still many unknowns regarding the measurement, analysis, and exploitation of natural fluorescence, which make necessary the development of appropriate models and campaigns to assess, using ground based measurements, the remaining technical, instrumental and basic scientific fluorescence issues. In this project validation data to test the integrated leaf-canopy fluorescence and reflectance models will exploit the team's experience with instruments that have been developed since 1995 for passive Chl fluorescence remote sensing: these are based on the atmospheric oxygen absorption bands, a single channel instrument measuring at $760 \mathrm{~nm}$ [4] and a multichannel instrument measuring at 760 and $687 \mathrm{~nm}$ have been 
developed [5]. Both instruments are operational and have been tested in several campaigns [6].

\section{PROJECT TASKS}

\section{A. Review for existing vegetation fluorescence models, selection of an appropriate model}

We shall analyse and discuss the fluorescence reflectancetransmittance model approaches as currently documented in the literature. Based on the findings a model appropriate to be used in conjunction with radiative transfer canopy models shall be proposed. In addition, we shall propose an appropriate radiative transfer canopy model to be used for this study

\section{B. Analysis of the fluorescence model, model advancement}

Following the model selection, a range of simulations shall be performed using realistic input variables. Model outputs shall be validated on the basis of consistency checks and existing measurements documented in the literature. Any necessary model improvements shall be made to account for discrepancies between model output and measurements.

\section{Upscaling/integration of a leaf model into a canopy model}

We shall integrate a validated leaf fluorescence model into an existing radiative transfer (RT) canopy model to construct a new RT canopy model accounting also for the fluorescence signal.

\section{Model validation using campaign data and comparison with indirect retrieval methods}

The new canopy model shall be validated by the use of existing and new measurements. A primary dataset will be that resulting from the ESA experiment SIFLEX-2002 (Solar Induced Fluorescence Experiment), in combination with the EU-supported activity SIBOFLEX (Solar Induced Boreal Forest Fluorescence Experiment). In addition, DAISEX campaign data (AD2), which also will be made available by the Agency, shall be used for model simulations to estimate the fluorescence signal level over canopies under different viewing conditions. Results will be compared with indirect fluorescence retrieval methods as developed for hyperspectral data cubes.

\section{CANOPY FLUORESCENCE MODELING APPROACHES}

Fluorescence emission at the canopy level can be considered as the integration of fluorescence emission processes at different levels from molecule to leaf and from leaf to canopy. In consequence, the leaf-canopy fluorescence model should integrate three sub-models corresponding to the three levels of organization (Fig. 1). In this approach, three different coupled models will be considered:

1. A physiological model, which predicts chlorophyll fluorescence quantum yield and spectral distribution of fluorescence on the basis of stress conditions (light quality and intensity during the day, stomatal conductance, etc.).
2. A biophysical leaf fluorescence-reflectancetransmittance model which accounts, at the leaf scale, for the spectral distribution of the incident light and the leaf chlorophyll content to simulate the fluorescence emission bands and their relative strengths, as well as the transmitted and reflected radiance spectra.

3. A canopy radiative transfer model which integrates fluorescence at the leaf level to compute canopy fluorescence emission.

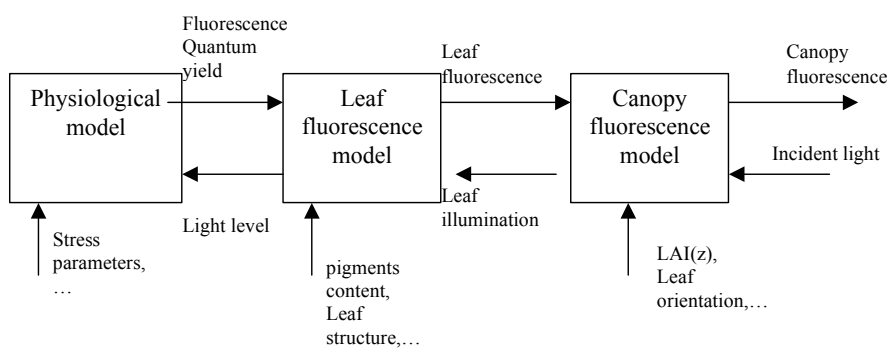

Figure 1. Modular representation of the leaf-canopy fluorescence model.

\section{A. Leaf level fluorescence and physiological model}

A broad leaf model will be developed according to existing work and to recent knowledge on chlorophyll a fluorescence. Since needle-shaped leaves would require a specific approach, because of the short time dedicated to this task, we will limit the study to one model. The latter implies an ability to model the fluorescence quantum yield efficiency with which the energy of a photon absorbed by this pigment is emitted as fluorescence photon as a function of the leaf physiological state (light intensity, stress, etc.).

Leaf models are essential to understand how electromagnetic radiation interacts with leaf elements, and also to directly relate observed optical properties to leaf biophysical attributes. Although several leaf models have been developed to relate leaf biochemistry and scattering parameters to leaf reflectance and transmittance signatures, only a few are formulated to specifically include the chlorophyll fluorescence signal. PROSPECT and LIBERTY, the two most popular models to simulate the reflectance and transmittance of broadleaves and needles respectively, do not include fluorescence, however they (particularly PROSPECT) have been validated on several datasets and are widely used in remote sensing.

Recent modeling of chlorophyll fluorescence at the leaf level for broad band illumination has been carried out with the FRT (Fluorescence-Reflectance-Transmittance) model [7] which used an adaptation of a doubling method solution of the Kubelka-Munk equations [10] and a two Gaussian representation of the fluorescence emission peaks near $690 \mathrm{~nm}$ and $740 \mathrm{~nm}$. In another approach, the SLOP (Stochastic model for Leaf Optical Properties) model [8] has been used to investigate the chlorophyll fluorescence contribution to the observed reflectance signature.

Current efforts are focused on developing a leaf model with the robustness of and accuracy of PROSPECT to simulate leaf 
reflectance and transmittance with the added attributes of FRT or SLOP to simulate the red and far red fluorescence emissions as a function of light intensity and quality incident on a particular leaf, accounting as well for the fluorescence quantum yield as a function of environmental variables.

\section{B. Canopy level fluorescence model}

The literature on canopy level fluorescence models presents only the model of Olioso et al. [9] and the FLSAIL model [10].

The model of Olioso et al. assumes a Lambert-Beer law attenuation of light flux inside the canopy, and considers only direct solar flux and the generated radiance in the direction of view. Although this model has the flexibility, useful in crops for example, of allowing a vertical gradient of the leaf chlorophyll content, it suffers a significant disadvantage for fluorescence simulation in that a dependence of leaf fluorescence efficiency on the light level incident on leaves is not accommodated.

The FLSAIL model shares its description of canopy geometry with the one applied in SAILH [12]. This model is identical to the original SAIL model, but with an extension to include the hot spot effect in the single scattering contribution. In FLSAIL all light interactions are modelled similarly to SAILH, but fluorescence contributions are added. Also, a different method of solution has been chosen and in FLSAIL the doubling algorithm is used for solution rather than an analytic solution. Other properties of this model are that the canopy layer is assumed to be homogeneous, that no dependence of leaf fluorescence on the incident light level is assumed, and that it was developed with the application to laser-induced fluorescence in mind. In FLSAIL a simple leaf level fluorescence model is included that is based on the Kubelka-Munk equations, extended for fluorescence. This leaf level sub-model is also solved by using the doubling algorithm.

Because this project focuses on simulations of solarinduced fluorescence, its scope must be extended beyond FLSAIL, which was only intended for modelling of laserinduced canopy fluorescence. A prerequisite for realistic modelling of passive fluorescence is a representative spectral distribution of the incident sun- and skylight. This could be accomplished by using an atmospheric radiative transfer model. Coupling the canopy model to an atmospheric model would have the additional advantage that not only surface fluorescence could be simulated, but also top-of-atmosphere radiance levels.

Another issue under consideration is whether or not to incorporate the dependence of fluorescence efficiency on the light level inside the canopy. Such dependence has a significant impact on the modelling concept that one can choose. For instance, it would mean that the canopy layer could no longer be assumed to be homogeneous, as the optical properties of the leaves would vary in the vertical direction. As a consequence one may need to replace the fast doubling algorithm by the much slower adding method.

\section{CONCLUSIONS}

The project goal is to develop and validate a canopy fluorescence model which can serve the needs of the community in improving the science basis of assessing the technical feasibility of a space mission to measure the solar induced fluorescence signal. This is recognized as a challenging task with a number of unanswered questions to be addressed in the coming months.

\section{REFERENCES}

[1] A. Ounis, "Télédétection de la fluorescence des couverts végétaux induite par laser : application des techniques de corrélation temporelle microseconde et nanoseconde" [Ph.D.], Université de Paris-Sud, 2001.

[2] M.-P. Stoll, A. Court, K. Smorenburg, H. Visser, L. Crocco, J. Heilimo, A. Honig, "FLEX-Fluorescence Explorer - a space mission for scrdeening vegetated areas in the Fraunhofer lines", Europto Conference on Remote Sensing for Earth Science Applications, Florence, Italy, SPIE Vol. 3868, 108-119, 1999.

[3] K. Smorenburg, G. Courreges-Lacoste, M. Berger, C. Buschman, A. Court, U. del Bello, G. Langsdorf, H. Lichtenhaler, C. Sioris, M.-P., Stoll, H. Visser, "Remote sensing of solar induced fluorescence of vegetation", in Remote Sensing of Agriculture, Ecosystems, and Hydrology III, M. Owe and G. D’Urso, Eds., Proc. of SPIE Vol. 4542, 178-190, 2002 .

[4] I. Moya, L. Camenen, G. Latouche, C. Mauxion, S. Evain, and Z. Cerovic, "An instrument for the measurement of sunlight excited plant fluorescence", in Photosynthesis: Mechanisms and Effects, G. Gorab, Ed., Kluwer Acad. Pub, Dordrecht, pp. 4265-4270, 1998.

[5] S. Evain, L. Camenen and I. Moya, "Three channels detector for remote sensing of chlorophyll fluorescence and reflectance from vegetation", in 8th Int. Symp.: Physical Measurements and Signatures in Remote Sensing, M. Leroy, Ed., Aussois, France, CNES, 395-400, 2001.

[6] S. Evain, A. Ounis, F. Baret, Y. Goulas, J. Louis, J-M. Ducruet, Z. Cerovic and I. Moya, "Passive vegetation fluorosensing using atmospheric oxygen absorption bands", Recent Advances in Quantitative Remote Sensing, 16-20 September 2002, Valencia, Spain, J. Sobrino, Ed.

[7] P. Zarco-Tejada., J. Miller, G. Mohammed, and T. Noland, "Chlorophyll fluorescence effects on vegetation apparent reflectance: I. Leaf-level measurements and model simulation", Remote Sens. Environ., 74(3):582-595, 2000

[8] S. Maier, W. Lüdeker, K. Günther, "SLOP: A revised version of the stochastic model for leaf optical properties", Remote Sens. Environ., 68(3):273-280, 1999.

[9] A. Olioso, M. Méthy, and B. Lacaze, "Simulation of canopy fluorescence as a function of canopy structure and leaf fluorescence", Remote Sens. Environ. 41: 239-247, 1992.

[10] A. Rosema, W. Verhoef, J. Schroote, and J. Snel, "Simulating lightcanopy interaction in support of laser-induced fluorescence measurements", Remote Sens. Environ. 37: 117-130, 1991.

[11] W. Verhoef, "Light scattering by leaf layers with application to canopy reflectance modeling: the SAIL model", Rem. Sens. of Env. 16: 125$141,1984$.

[12] W. Verhoef, "Theory of radiative transfer models applied in optical remote sensing of vegetation canopies", $\mathrm{PhD}$ Thesis, Agricultural University Wageningen, $310 \quad$ pp. 1998. 